\title{
Watt-scale 50-MHz source of single-cycle waveform-stable pulses in the molecular fingerprint region
}

\author{
T. P. Butler, ${ }^{1}$ (i) D. Gerz, ${ }^{1,2}$ C. Hofer, ${ }^{1,2}$ J. Xu, ${ }^{1}$ (1) C. Gaida, ${ }^{3}$ (이 T. Heuermann, ${ }^{3,4}$ M. Gebhardt, ${ }^{3,4}$ \\ L. Vamos, ${ }^{2}$ W. Schweinberger, ${ }^{2,5}$ J. A. Gessner, ${ }^{1,2}$ T. Siefke, ${ }^{3,6}$ M. Heusinger, ${ }^{3}$ U. Zeitner, ${ }^{3,7}$ \\ A. Apolonski, ${ }^{1,2}$ N. Karpowicz, ${ }^{1}$ J. Limpert, ${ }^{3,4,7}$ F. Krausz, ${ }^{1,2}$ And I. Pupeza ${ }^{1,2, *}$ (1) \\ ${ }^{1}$ Max Planck Institute of Quantum Optics, Hans-Kopfermann-Str. 1, 85748 Garching, Germany \\ ${ }^{2}$ Ludwig Maximillians University Munich, Am Coulombwall 1, 85748 Garching, Germany \\ ${ }^{3}$ Institute of Applied Physics, Abbe Centre of Photonics, Friedrich-Schiller Universität Jena, Albert-Einstein-Str. 15, 07745 Jena, Germany \\ ${ }^{4} \mathrm{Helmholtz-Institute}$ Jena, Frobelstieg 3, 07743 Jena, Germany \\ ${ }^{5}$ King Saud University, Department of Physics and Astronomy, Riyadh 11451, Saudi Arabia \\ ${ }^{6}$ Physikalisch-Technische Bundesanstalt, Bundesallee 100, 38116 Braunschweig, Germany \\ ${ }^{7}$ Fraunhofer Institute for Applied Optics and Precision Engineering, Albert-Einstein-Str. 7, 07745 Jena, Germany \\ ${ }^{*}$ Corresponding author: ioachim.pupeza@mpq.mpg.de
}

Received 15 January 2019; revised 27 February 2019; accepted 1 March 2019; posted 4 March 2019 (Doc. ID 357850); published 27 March 2019

\begin{abstract}
We report a coherent mid-infrared (MIR) source with a combination of broad spectral coverage $(6-18 \mu \mathrm{m})$, high repetition rate $(50 \mathrm{MHz})$, and high average power $(0.5 \mathrm{~W})$. The waveform-stable pulses emerge via intrapulse differencefrequency generation (IPDFG) in a GaSe crystal, driven by a 30-W-average-power train of 32-fs pulses spectrally centered at $2 \mu \mathrm{m}$, delivered by a fiber-laser system. Electro-optic sampling (EOS) of the waveform-stable MIR waveforms reveals their single-cycle nature, confirming the excellent phase matching both of IPDFG and of EOS with 2- $\mu \mathrm{m}$ pulses in GaSe. () 2019 Optical Society of America
\end{abstract}

https://doi.org/10.1364/OL.44.001730

Provided under the terms of the OSA Open Access Publishing Agreement

Coherent mid-infrared (MIR) light sources have shown promise in the study of a wide range of resonant light-matter interactions, most notably in the study of ro-vibrational molecular transitions and dynamics [1], as well as the investigation of low-energy phonon dynamics [2], charge transport in semiconductors [3], and non-linear and high-field interactions [4]. As such, the development of both suitable light sources and appropriate detection techniques is an important step toward the improvement of the sensitivity and precision of measurements, especially in applications such as biomedical diagnostics [5] and chemical/trace gas monitoring [6,7]. Recent advances in nearinfrared (NIR) femtosecond laser technology have provided a viable route towards table-top broadband ultrafast MIR sources via nonlinear processes such as parametric amplification or difference frequency generation (DFG) [6-12]. In particular, the frequency down-conversion technique of intra-pulse DFG (IPDFG) has emerged as a relatively simple way to create MIR pulse trains that combine several properties highly desirable for molecular spectroscopy $[7,10-12]$. IPDFG, which generates a
MIR idler signal through phase matched mixing of spectral components within a broadband driving pulse, ensures a passively waveform-stable output. This enables electro-optic sampling (EOS) to detect the generated electric field, transfer the detection to the NIR spectral region where low-noise semiconductor photodetectors offer increased performance over cooled MIR detectors [13] used in conventional Fourier transform infrared (FTIR) spectroscopy, and allows for ultrasensitive [14] and broadband [15,16] detection across the entire molecular fingerprint region.

The major limitation of IPDFG-based MIR sources so far has been the severe trade-off between bandwidth and efficiency in the nonlinear conversion process. Similarly, where high fieldstrengths are desirable, drivers are typically limited to $\mathrm{kHz}$ repetition rates [17]. In multi-MHz repetition rate systems, NIR-to-MIR conversion efficiencies on the order of $0.1 \%$ have been demonstrated for 1- $\mu \mathrm{m}$ [10], 1.5- $\mu \mathrm{m}$ [7], and 2- $\mu \mathrm{m}$ [11] sources. Very recently, IPDFG at a $1.25-\mathrm{MHz}$ repetition rate with a conversion efficiency of $1.8 \%$ driven by $2-\mu \mathrm{m}, 16-\mathrm{fs}$ pulses, generating a spectrum from 7.3 to $16.5 \mu \mathrm{m}$ (at $-10 \mathrm{~dB}$ ) and a record power of $470 \mathrm{~mW}$ was reported [12]. Driving pulses at $2-\mu \mathrm{m}$ can offer increased powers due to a lower quantum defect and lower photon energy, enabling the use of smaller-bandgap crystals with higher nonlinear coefficients [18]. At the same time, longer driving wavelengths increase the phase matched bandwidth by improving the group velocity matching between NIR and MIR pulses [8]. While the previous results were obtained with high driving pulse energies $(24 \mu \mathrm{J})$, they show the existence of favorable phase matching conditions for high-efficiency, broad-bandwidth IPDFG using GaSe as a nonlinear medium. These results show the possibility to achieve high-conversion efficiency and broad phasematching via IPDFG using GaSe as a nonlinear medium, albeit 
using a large pulse energy and $1.25-\mathrm{MHz}$ repetition rate. Here, we further investigate the suitability of $2-\mu \mathrm{m}$ driven IPDFG to assess whether this conversion efficiency can be maintained at the high repetition rates typically needed for frequency-comb spectroscopy $[6,7]$ and also characterize the temporal profile of the MIR pulse, which is essential for field-resolved spectroscopy $[19,20]$.

In this Letter, we present a source which combines a watt-scale average power $(0.5 \mathrm{~W})$, super-octave bandwidth (6-18 $\mu \mathrm{m})$, single-cycle pulse duration (43 fs), waveform stability, and high repetition rate $(50 \mathrm{MHz})$. Our novel MIR source is based on $2-\mu \mathrm{m}$ fiber laser technology, enabling both generation and field-sensitive detection of phase-stable MIR pulses. These MIR transients, generated via IPDFG of 30-W average power, 32-fs pulses in a GaSe crystal, have been characterized using EOS with few-cycle, watt-level $2-\mu \mathrm{m}$ sampling pulses. Compression of the field to a single-cycle duration has been demonstrated, with the possibility to generate absolute field strengths in excess of $1 \mathrm{MV} / \mathrm{cm}$ at a $50-\mathrm{MHz}$ rate. Compared to current state-of-the-art broadband MIR sources, our table-top system provides a significantly higher spectral brilliance across its entire bandwidth than current 3rd-generation facility-scale synchrotron sources [21], while maintaining a high degree of temporal and spatial coherence and close to bandwidth limited temporal compression.

The experimental setup of the $2-\mu \mathrm{m}$ femtosecond frontend is shown in Fig. 1. The amplifier chain was seeded by a train of $50-\mathrm{MHz}$ pulses from a fiber laser. This commercial source (Menlo C-Fiber) generated 2- $\mu \mathrm{m}$ pulses from a 1550-nm Er:fiber seed oscillator which was amplified and Raman shifted in a subsequent nonlinear fiber. Stretched pulses with a center wavelength of $1965 \mathrm{~nm}$ were subsequently amplified in two thulium-doped fibers boosting the average power of the pulse train to a maximum of $100 \mathrm{~W}$ [22]. Due to absorption by ambient water vapor, the entire high-power free-space beam path was housed in vacuum chambers at a pressure of $<1 \mathrm{mbar}$, avoiding thermal lensing and preserving the beam quality [23]. At an output power of $40 \mathrm{~W}$, the compressed 250 -fs pulse train from the CPA was split between 2 channels, and further compressed nonlinearly in photonic crystal fibers (PCF) [24]. To achieve the high-average-power driving pulses required for efficient IPDFG, the first channel was compressed to 32-fs full width at half-intensity maximum (FWHM) using a $56-\mu \mathrm{m}$ mode-field diameter (MFD) solid-core silica PCF with a length

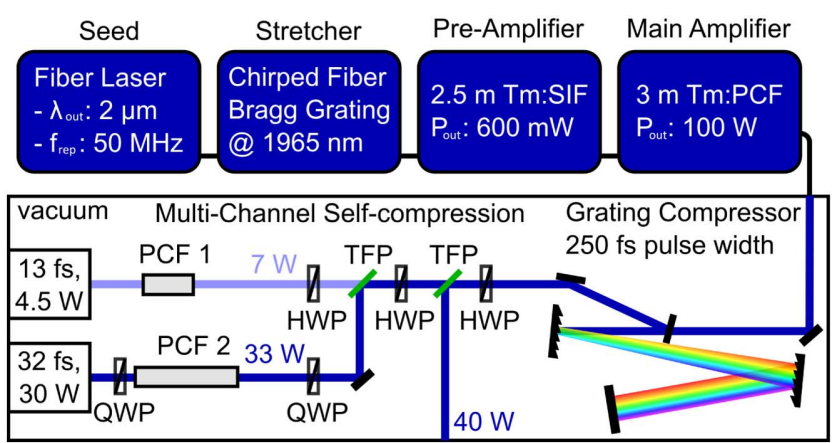

Fig. 1. Experimental schematic of the Tm:fibre-based CPA frontend. SIF: step index fiber; HWP: half wave plate; TFP: thin film polarizer; QWP: quarter wave plate; PCF 1: 13- $\mu \mathrm{m}$ MFD; PCF 2: 56- $\mu \mathrm{m}$ MFD.

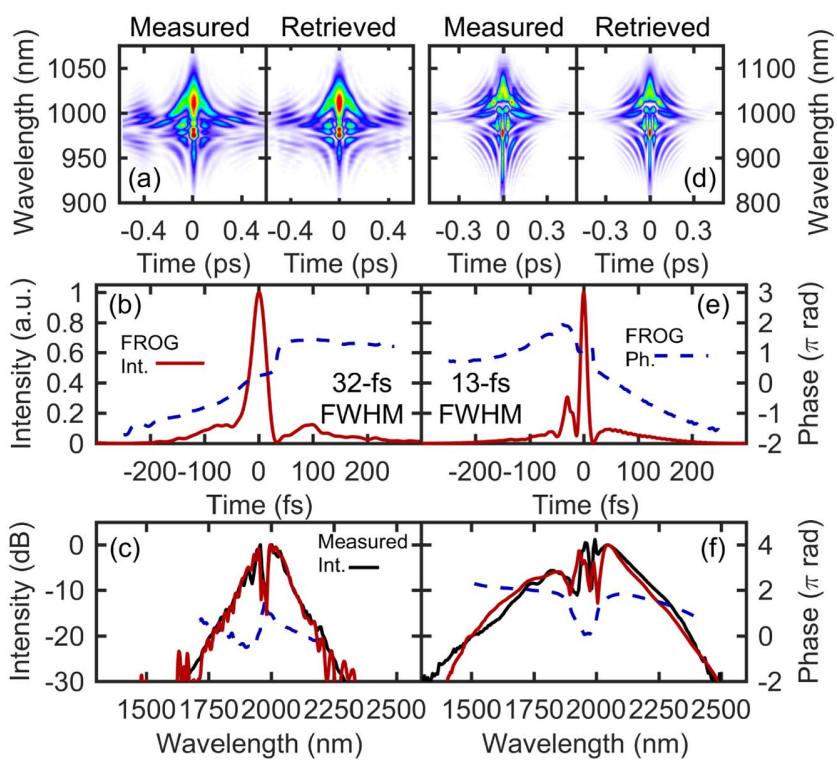

Fig. 2. (a) Measured [left] and retrieved [right] FROG spectrograms of the longer pulse PCF compression channel. (b) Retrieved FROG temporal intensity and phase. (c) Retrieved FROG spectral intensity and phase, compared to independently measured spectrum obtained using a NIR grating spectrometer. ( $\mathrm{d}-\mathrm{f})$ shows the same information as $(\mathrm{a}-\mathrm{c})$, this time measured for the shorter pulse PCF channel.

of $5.6 \mathrm{~cm}$. The self-compressed pulse width of 32-fs FWHM was measured with a home-built second-harmonic-generation frequency-resolved optical gating (SHG-FROG) instrument utilizing a $10-\mu \mathrm{m}$ thick BBO crystal, see Figs. 2(a)-2(c). For accurate temporal measurement of MIR waveforms, a second fiber self-compression stage was implemented in parallel for use as the EOS sampling pulse train. In this case, a smaller core PCF fiber (13- $\mu \mathrm{m}$ MFD and $2.3 \mathrm{~cm}$ long) was used to spectrally broaden the CPA driving pulses to a width of $700 \mathrm{~nm}$ at $-20 \mathrm{~dB}$ with $>60 \%$ efficiency while simultaneously temporally compressing the pulse to $\sim 2$ optical cycles (13-fs FWHM), see Figs. 2(d) $-2(\mathrm{f})$.

Figure 3 presents a schematic layout of the IPDFG and EOS setup. The 32-fs p-polarized pulse train was focused to a $162-$ $\mu \mathrm{m} 1 / e^{2}$-intensity spot diameter inside a 1 -mm-thick GaSe

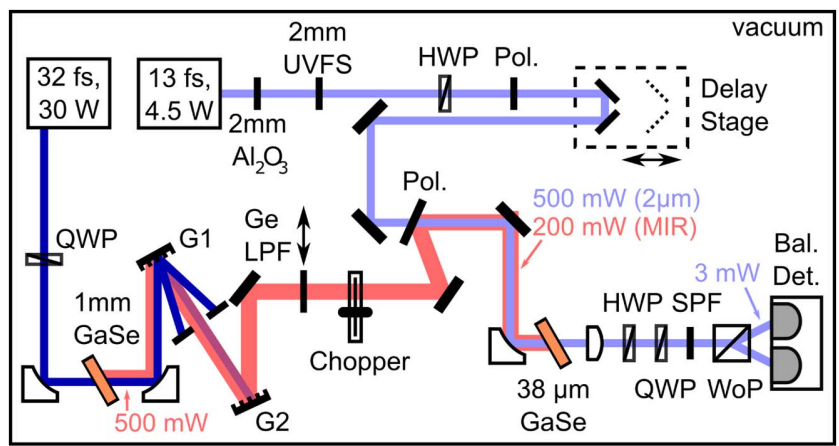

Fig. 3. Experimental setup of the IPDFG and EOS. G1, G2 are gold-coated silicon beamsplitter gratings. Ge LPF: coated 1-mm Ge longpass filter with 4.5 um cut-on. Pol.: wire grid polarizer; UVFS: UV fused silica; SPF: shortpass filter $1550 \mathrm{~nm}$. WoP: Wollaston prism; Bal. Det.: balanced InGaAs photodetector. 
crystal. Type-I phase matching was used with an external crystal angle of 38 degrees [12]. The resulting MIR light was measured with a wire-grid polarizer to be s-polarized and was collimated using a gold-coated off-axis parabola. Separation of the collinearly propagating MIR and driving signals was achieved by using custom-fabricated gold-coated silicon diffraction gratings [25]. These gratings were designed to have a high diffraction efficiency at $2 \mu \mathrm{m}$, while retaining a large zeroth-order reflection coefficient for the MIR wavelengths. Three gratings were used in series in order to suppress the power of the driving pulses by $30 \mathrm{~dB}$. Compared to other coated or transmissive filtering optics, diffraction gratings offer an exceptional thermal stability and impart no chromatic dispersion on the propagating MIR pulses. A reflective $\mathrm{ZnSe}$ wire-grid polarizer was used to combine the MIR pulses with the $2-\mu \mathrm{m}$ sampling pulses in an orthogonal polarization state. Use of a reflective beamsplitter and recombiner meant that no transmissive elements were present in the MIR beam path, enabling a direct measurement of the MIR pulse as it exits the IPDFG crystal. After undergoing mixing in the GaSe crystal, at least $498 \mathrm{~mW}$ of MIR power was generated after the exit face of the crystal, estimated by accounting for the loss from subsequent mirrors and optics. Considering the power lost due to reflection from the front face of the crystal, a total NIR-to-MIR conversion efficiency of $2.0 \%$ was achieved with a peak intensity at the focus of $75 \mathrm{GW} / \mathrm{cm}^{2}$.

For lock-in detection of the electric field of the broadband MIR transients, the beam was mechanically chopped at a frequency of $7 \mathrm{kHz}$. The short p-polarized probe was combined with the MIR pulse train by transmission through a $\mathrm{ZnSe}$ wire grid polarizer. Dispersion of the probe pulse was compensated using bulk fused silica and sapphire plates, ensuring a 20 -fs FWHM pulse, which was spatially overlapped with the MIR beam and focused into a 38- $\mu \mathrm{m}$ thick GaSe crystal. Sumfrequency generation (SFG) of the two pulses results in an effective polarization rotation of the probe pulse, which is directly proportional to the MIR electric field [26]. Scanning of the probe pulse delay allows for detection of the MIR field. The thin detection crystal ensured good phase matching across the entire MIR spectrum. The measured EOS trace of the MIR pulse is shown in Fig. 4(a). While a clear chirp of the waveform can be observed due to the dispersion-free nature of the beam-path, it is inferred that this chirp is intrinsic to either the MIR wave passing through the nonlinear generation crystal or to the response of EOS detection. Numerical modelling of the EOS detection allows for retrieval of the input MIR electric field through independent calculation of the EOS instrument response function [15]. The retrieved field, also shown in Fig. 4(a), excludes the latter as the source of the chirp. Figure 4(b) compares the spectrum of the measured EOS trace to the retrieved EOS input spectrum as well as the independently measured spectrum captured with an FTIR spectrometer. The good agreement of the two spectra validates the calculation of the EOS response. Comparison of the EOS and FTIR spectra shows that phase matching in the EOS detection process slightly suppresses the signal at longer wavelengths, while enhancing detection for the shorter ones, as clearly observed in the chirped waveforms of Fig. 4(a).

For the study of both linear $[19,20]$ and nonlinear $[4,17]$ interactions, the confinement of the available spectrum to a short transient is essential. Dispersion compensation of pulses in this spectral range can be difficult due to a relatively small
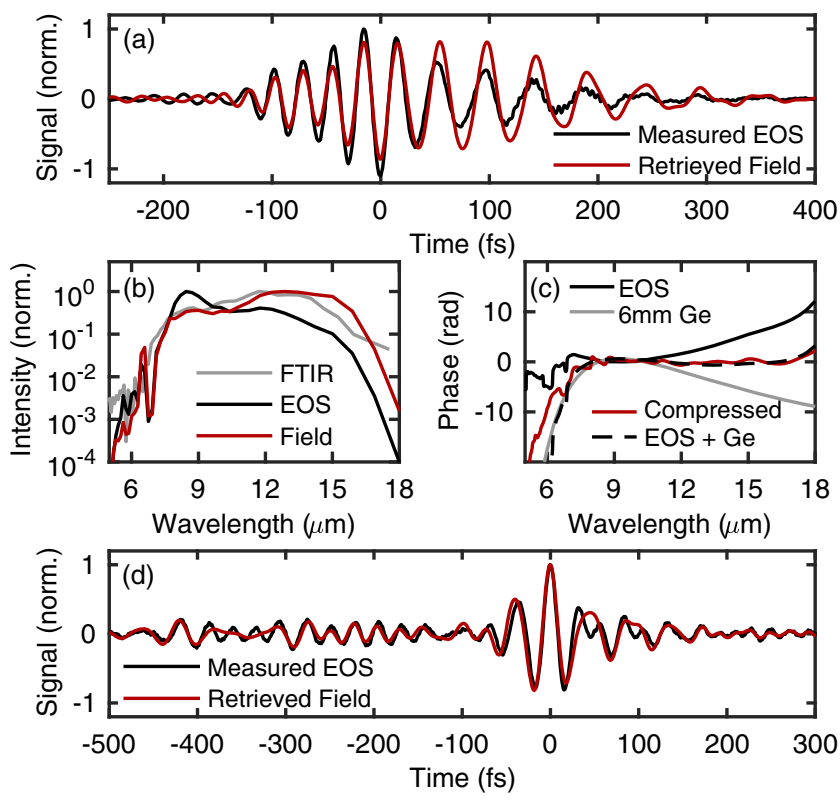

Fig. 4. (a) Measured EOS trace of MIR transient generated by IPDFG (black) and recovered MIR input field (red). (b) Measured EOS spectrum (black) (Fourier transform of the temporal waveform) compared with the independently measured FTIR spectrum (grey) and the recovered spectrum (red). (c) Spectral phase of the measured EOS pulse (black). Phase due to $6 \mathrm{~mm}$ of propagation through bulk germanium (grey). Sum of EOS phase and Ge (black dashed). Measured spectral phase after insertion of bulk Ge in the beam path (red). (d) Measured EOS after compression with bulk Ge (black) and the retrieved field (red) with a 43-fs intensity FWHM.

choice of suitable materials. Figure 4(c) shows the measured spectral phase of the pulse shown in parts (a) and (b). Also shown is the phase expected due to dispersion through $6 \mathrm{~mm}$ of bulk germanium. It is clear that in the longer wavelength part of the spectrum $(\sim 9-20 \mu \mathrm{m})$, germanium can provide suitable compensation for the generated IPDFG pulse [27]. Using this arrangement, we experimentally demonstrated compression of the MIR transient by performing EOS detection after transmitting the pulse through $6 \mathrm{~mm}$ of bulk germanium. Figure 4(c) shows the measured spectral phase, which agrees well with the numerical calculations. Figure $4(\mathrm{~d})$ shows the measured and retrieved field for the compressed pulse, with an intensity FWHM of $43 \mathrm{fs}$. This pulse duration is just 1.16 times the fundamental oscillation period of the central carrier field at $11.1 \mu \mathrm{m}$. In this case, the compression medium introduces some Fresnel reflection losses to the MIR beam. However, with a Brewster-angle compression plate and minimal power loss, the absolute field strength of the transient could exceed $4 \mathrm{MV} / \mathrm{cm}$. The generation of such field strengths at a $>10 \mathrm{MHz}$ repetition rate, combined with the single-cycle duration, make this source ideally suited to multi-dimensional spectroscopy and other pump-probe experiments. Along with high output power, the spectrum of the MIR idler covers a broad bandwidth, from 6 to $18 \mu \mathrm{m}(-30 \mathrm{~dB})$.

Figure 5(a) shows the calculated spectral brilliance [9] compared to two other commonly used MIR radiation sources, thermal and synchrotron (BESSY II IRIS beamline) [21]. The brilliance of our coherent MIR source is at least 2 orders of magnitude greater over $7-17 \mu \mathrm{m}$ and exceeds that of the 


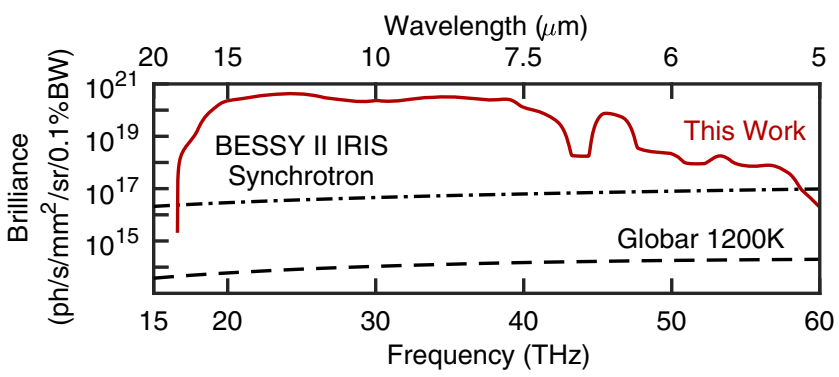

Fig. 5. Photon brilliance of the MIR spectrum calculated from the measured average power and retrieved EOS spectrum, compared with other common FTIR sources [21].

synchrotron over nearly two octaves (from 5 to $18 \mu \mathrm{m}$ ). The power spectral density of the source is greater than $1 \mathrm{~mW} / \mathrm{THz}$ across a large part of the spectrum $(20-40 \mathrm{THz})$, while the power per combline value exceeds $1 \mu \mathrm{W}$ in the 19 to $27.5 \mathrm{THz}$ region, with a peak of $2.2 \mu \mathrm{W}$ per combline. This value is more than 10 times larger than previously reported $2-\mu \mathrm{m}$ driven IPDFG sources with a peak value of $<70 \mathrm{nW}$ [11] and $<200 \mathrm{nW}$ [12] per combline and over 3 orders of magnitude greater than state-of-the-art $1.5-\mu \mathrm{m}$ driven MIR combs with $\sim 1 \mathrm{nW}$ per combline [7].

In conclusion, we have demonstrated a phase-stable, high-power $(0.5 \mathrm{~W})$, broad-band MIR source, covering the long-wavelength part of the MIR fingerprint region (550$2000 \mathrm{~cm}-1)$. Combined for the first time with a high repetition rate $(50 \mathrm{MHz})$, ultrashort pulse duration (43 fs), and fieldresolved EOS detection with high-power, $2-\mu \mathrm{m}$ pulses, this system presents a flexible, compact, and cost-effective platform for precision measurements across a wide gamut of applications. These results demonstrate the advantage of employing a $2-\mu \mathrm{m}$ based pump source over $1 \mu \mathrm{m}$ for IPDFG-based MIR generation, with higher efficiencies and broader phase matching. Even higher average powers and peak field strengths may be possible with further power scaling of the driver, leveraging advances in Tm:fibre CPA [28] or bulk-based nonlinear compression [29]. This may also offer the ability to use thinner generation media, boosting the bandwidth toward multi-octave terahertz spectra and even shorter sub-cycle transients [15] while retaining high output power. The ultrashort MIR pulse train is well suited for background-free time-resolved studies of molecular dynamics, corresponding in the frequency domain to a harmonic frequency comb with a record $\mu \mathrm{W}$-class power per combline. Finally, we have shown for the first time EOS detection of short MIR transients by means of a $2-\mu \mathrm{m}$ based sampling pulse, demonstrating favorable phase-matching conditions for a broad MIR band. A high average sampling power meanwhile benefits the efficiency of the frequency upconversion step in EOS detection [26]. These results establish $2-\mu \mathrm{m}$ fs technology as a new, viable route toward high-power, high-precision, field-resolved molecular vibrational metrology.

Funding. Munich-Centre for Advanced Photonics (MAP); Deutsche Forschungsgemeinschaft (DFG) (KR4768/1-1).

Acknowledgment. We thank Ronald Holzwarth and Michael Mei from Menlo Systems for their support with the custom seed laser.

\section{REFERENCES}

1. W. Demtröder, Molecular Physics (Wiley, 2005).

2. T. Kampfrath, L. Perfetti, F. Schapper, C. Frischkorn, and M. Wolf, Phys. Rev. Lett. 95, 187403 (2005).

3. R. Huber, F. Tauser, A. Brodschelm, M. Bichler, G. Abstrelter, and A. Leitenstorfer, Nature 414, 286 (2001).

4. C. Schmidt, J. Buhler, A.-C. Heinrich, J. Allerbeck, R. Podzimski, D. Berghoff, T. Meier, W. G. Schmidt, C. Reichl, W. Wegscheider, D. Brida, and A. Leitenstorfer, Nat. Commun. 9, 2890 (2018).

5. A. Barth and P. I. Haris, Biological and Biomedical Infrared Spectroscopy (IOS press, 2009).

6. A. V. Muraviev, V. O. Smolski, Z. E. Loparo, and K. L. Vodopyanov, Nat. Photonics 12, 209 (2018).

7. H. Timmers, A. Kowigly, A. Lind, F. C. Cruz, N. Nader, M. Silfies, G Ycas, T. K. Allison, P. G. Schunemann, S. B. Papp, and S. A. Diddams, Optica 5, 727 (2018).

8. F. Junginger, A. Sell, O. Schubert, B. Mayer, D. Brida, M. Marangoni, G. Cerullo, A. Leitenstorfer, and R. Huber, Opt. Lett. 35, 2645 (2010).

9. C. Manzoni, M. Forst, H. Ehrke, and A. Cavalleri, Opt. Lett. 35, 757 (2010).

10. I. Pupeza, D. Sanchez, J. Zhang, N. Lilienfein, M. Seidel, N. Karpowicz, T. Paasch-Colberg, I. Znakovskaya, M. Pescher, W. Schweinberger, V. Pervak, E. Fill, O. Pronin, Z. Wei, F. Krausz, A. Apolonski, and J. Biegert, Nat. Photonics 9, 721 (2015).

11. J. Zhang, K. F. Mak, N. Nagl, M. Seidel, D. Bauer, D. Sutter, V. Pervak, F. Krausz, and O. Pronin, Light: Sci. Appl. 7, 17180 (2018).

12. C. Gaida, M. Gebhardt, T. Heuermann, F. Stutzki, C. Jauregui, J. Antonio-Lopez, A. Schulzgen, R. Amezcua-Correa, A. Tunnermann, I. Pupeza, and J. Limpert, Light: Sci. Appl. 7, 94 (2018).

13. A. Rogalski, Opto-Electron. Rev. 20, 279 (2012).

14. C. Riek, D. V. Seletskiy, A. S. Moskalenko, J. F. Schmidt, P. Krauspe, S. Eckart, S. Eggert, G. Burkar, and A. Leitenstorfer, Science 350, 420 (2016).

15. C. Kubler, R. Huber, S. Tubel, and A. Leitenstorfer, Appl. Phys. Lett 85, 3360 (2004)

16. S. Keiber, S. Sederberg, A. Schwarz, M. Trubetskov, V. Pervak, F. Krausz, and N. Karpowicz, Nat. Photonics 10, 159 (2016).

17. M. Knorr, J. Raab, M. Tauer, P. Merkl, D. Peller, E. Wittmann, E. Riedle, C. Lange, and R. Huber, Opt. Lett. 42, 4367 (2017).

18. V. Petrov, Prog. Quant. Electron. 42, 1 (2015).

19. I. Pupeza, M. Huber, W. Schweinberger, M. Trubetskov, S. A. Hussain, O. Pronin, L. Vamos, O. Pronin, F. Habel, V. Pervak, N. Karpowicz, E. Fill, A. Apolonski, M. Zigman, A. M. Azzeer, and F. Krausz, Conference on Lasers and Electro-Optics Europe European Quantum Electronics Conference (CLEO/Europe-EQEC) (IEEE, 2017), p. 8086921.

20. I. Pupeza, et al., "Field-Resolved Infrared Spectroscopy of Biofluids" (to be published).

21. L. Puskar, J. Large-Scale Res Facil. 2, A95 (2016).

22. C. Gaida, T. Heuermann, M. Gebhart, E. Shestaev, T. P. Butler, D. Gerz, N. Lilienfein, P. Sulzer, M. Fischer, R. Holzwarth, A. Leitenstorfer, I. Pupeza, and J. Limpert, Opt. Lett. 43, 5178 (2018).

23. M. Gebhart, C. Gaida, F. Stutzki, S. Hadrich, C. Jauregui, J. Limpert, and A. Tunnermann, Opt. Express 23, 13776 (2015).

24. C. Gaida, M. Gebhardt, F. Stutzki, C. Jauragui, J. Limpert, and A. Tunnermann, Opt. Lett. 40, 5160 (2015).

25. D. Gerz, W. Schweinberger, T. P. Butler, T. Siefke, M. Heusinger, T. Amotchkina, V. Pervak, U. Zeitner, and I. Pupeza, Opt. Lett. (to be published).

26. G. Gallot and D. Grischkowsky, J. Opt. Soc. Am. B 16, 1204 (1999).

27. D. Brida, M. Marangoni, C. Manzoni, S. De Silvestri, and G. Cerullo, Opt. Lett. 33, 2901 (2008).

28. C. Gaida, M. Gebhardt, T. Heuermann, F. Stutzki, C. Jauregui, and J. Limpert, Opt. Lett. 43, 5853 (2018).

29. K. Fritsch, M. Poetzlberger, V. Pervak, J. Brons, and O. Pronin, Opt. Lett. 43, 4643 (2018). 